\title{
L'ÉTAT EST-IL EN MESURE D'INFLÉCHIR LE «SORT» D'UNE LANGUE?
}

\begin{abstract}
Kramer Nataliya, L'État est-il en mesure d'infléchir le «sort» d'une langue? [Can a state influence the "fate" of a language?]. Studia Romanica Posnaniensia, Adam Mickiewicz University Press, Poznań, vol. XXX: 2003, pp. 95-106. ISBN 83-232-1270-8. ISSN 0137-2475.

The author ponders in her article - based mainly on the example of the Norwegian language - whether state's interference through its specialised organs may influence the development and formation of language norms. In conclusion the author claims that only the combination of "Ianguage management approach" and "free enterprise style" may give satisfactory results in managing problems of the language.
\end{abstract}

\section{INTRODUCTION}

Le monde contemporain se caractérise par l'universalisation des problèmes et des conflits linguistiques. Les débats publics sur la normalisation des langues, les réformes linguistiques, l'adoption des lois sur les langues, la création des comités de terminologie, tous ces phénomènes, disparates à première vue, ont un dénominateur commun: ils témoignent de l'accroissement du niveau de la conscience sociale en ce qui concerne les langues. Cet état de choses est, à son tour, en corrélation avec la logique du développement de la linguistique, qui tend, de plus en plus, à pénétrer dans de nouveaux domaines de la connaissance tels l'histoire et/ou la philosophie (par ex.: la philosophie herméneutique' de H.-G. Gadamer), l'informatique (par ex.: «Linguistique et informatique», le cours universitaire), le cinéma (les films de J.-L. Godard), pour n'en mentionner que quelques-uns. Il en est de même pour le pouvoir: comme l'affirme le philosophe français Michel Foucault, le

'Herméneutiquc (du gr. hermêneuticos - j'interprète), art et théorie de l'interprétation des textes, en se fondant sur des critères objectives (sens grammatical des mots et leurs variations déterminées par les circonstances historiques) ou sur les critères subjectives (intentions des auteurs) (DPh 1980: 224). 
«succès des formes modernes de domination réside dans la dispersion ( $c^{*}$ est nous qui soulignons) du pouvoir» (Dirk et al. 1994: 8).

Tandis que l'opinion publique assume que le pouvoir n'est exercé que par un État (d'où la perception négative du pouvoir), pour Foucault l'exercice du pouvoir n'est pas uniquement l'apanage d'un État. Le pouvoir, apolitique et désinstitutionnalisé, est présent dans l'ordre le plus quotidien et dans les rites les plus solennels («it [power] cornes from everywhere», Foucault 1978: 93): dans le travail, dans la rue, dans la pédagogie, dans les rapports familiaux, même dans les relations amoureuses. Le pouvoir fait partie intégrale des relations sociales, parce que chacun de nous exerce et subit le pouvoir simultanément.

On peut, à ce moment-là, construire le syllogisme qui suit:

- le pouvoir fait partie des relations sociales;

- les relations sociales se réalisent principalement par le biais des moyens linguistiques;

d'où,

- le pouvoir peut se réaliser par des moyens linguistiques.

En d'autres mots: on peut dominer par le biais d'une langue. Une langue, et surtout une langue standardisée (cf. ci-dessous), est une des importantes préoccupations de l'État, car elle est l'instrument de la communication avec les citoyens et un moyen efficace d'exercer un certain contrôle sur eux. On revient donc à la question de la régulation de l'activité linguistique, ou, en d'autres mots, de la planification ${ }^{2}$ ou de l'anténagement linguistique.

\section{L'AMÉNAGEMENT LINGUISTIQUE EST-IL VRAIMENT LINGUISTIQUE?}

\subsection{LA LANGUE, LA SOCIÉTÉ ET LE LINGUISTE}

Toute démarche visant la régulation des situations linguistiques a, à l'origine, d'après J.-Cl. Corbeil, le même état de choses: «la diversité linguistique au sein d'un même pays, la concurrence entre les langues ou les variantes de la même langue au sein des institutions et enfin la conscience de cette concurrence et l'intention collective de l'ordonner, voire de l'apaiseny (Corbeil 1980: 113). Selon T. Bull (Bull 1993: 29), l'aménagement linguistique est la réponse à un problème social, qui est senti plus fortement par certains groupes sociaux que par d'autres.

${ }^{2}$ Le terme est chargé d'une connotation péjorative, au dire de J-Cl. Corbeil (Corbeil 1980: 9), car il suggère «une intervention dirigiste, de type bureaucratique». 
Comme l'affirme F. Karam (Karam 1974: 108), «sans égard au type d'aménagement linguistique, dans presque tous les cas, le problème linguistique à résoudre [...] est étroitement lié avec la situation politique, économique, scientifique, sociale, culturelle et/ou religieuse». En outre, «l'aménagement linguistique est généralement réalisé pour atteindre les objectifs non-linguistiques tels la protection du consommateur, l'échange scientifique, l'intégration nationale, le contrôle politique, le développement économique, la création de nouvelles élites ou la préservation des anciennes, la pacification ou cooptation des groupes minoritaires, la mobilisation massive des mouvements nationaux et politiques» (Cooper 1983: 34-35). Bref, l'aménagement linguistique, comme tout aménagement, présuppose la volonté d'orienter le développement d'une langue dans une direction désirée. Est-il possible de changer le cours d'une langue, quand, selon certains linguistes, «le changement linguistique est un processus mystérieux et abstrait, qui semble n'être effectué par aucun organisme»? (Cameron 1990: 90). Les formulations de ce type, à notre avis, ont perdu leur caractère de postulats irréfutables. Prenons un autre argument du même ordre tel «la langue reflète la société», qui implique qu'une langue n'est que le reflet d'un changement social plus profond. Pense-t-on, par exemple, que l'accès des femmes aux fonctions jadis réservées aux hommes (au Québec en particulier) amènerait automatiquement, sans aucun débat sur le sexisme, sans aucune régulation linguistique, à l'implantation des formes féminisées? Nous tenons donc à souligner ici que les structures sociales sont à tel point inextricablement imbriquées avec les structures linguistiques, que le changement linguistique est lui-même un changement social. Et, comme les autres institutions sociales ont été changées par l'aménagement extérieur (Haugen 1966: 297), la langue peut aussi être changée par une influence externe.

L'activité professionnelle d'un linguiste qui traite les questions du fonctionnement social d'une langue ( $\mathrm{y}$ compris l'analyse des situations linguistiques et les jugements normatifs) se situe à mi-chemin entre une science (impartiale, descriptive, objective) et des batailles politico-idéologiques (émotives, évaluatives, subjectives). Le problème est celui d'un choix judicieux, car étant inévitablement dépendant des institutions du pouvoir et de l'idéologie dominante, un chercheur fait toujours face à plusieurs dilemmes. De l'un, «langues venaculaires vs langues véhiculaires»: appartient-il aux spécialistes de la langue de «rendre un verdict» favorisant la survivance (ou le déclin) des premières ou la diffusion triomphante des secondes? $?^{3}$ De l'autre, «objectivité vs subjectivisme»: l'aménagement linguistique, qui n'est pas exempt d'émotivité et d'énonciations personnelles du linguiste, est-il justifié, objectif et scientifique?

${ }^{3}$ On pourrait, dans ce cas-là, soulever la question du choix d'une langue internationale, mais cette question scmble avoir ćté résolue au cours du temps en faveur de l'anglais. 


\subsection{LE CARACTÈRE DU SIGNE LINGUISTIQUE}

Par rapport aux considérations antérieures, il nous semble pertinent de faire état de certains points de repère de la théorie de F. de Saussure, qui rejetait toute possibilité d'influencer une langue (c'est-à-dire un système de rapports entre signes, accepté par une communauté linguistique). Plus précisément, le système de signes fonctionne en vertu d'une sorte de convention sociale, parce que la même association «son pensée» se fait dans le cerveau de tous les sujets parlants. Une fois qu'une telle convention existe, il faut qu'elle soit respectée pour que le système puisse fonctionner. Les signes sont arbitraires: il n'y a pas de lien «naturel» entre le signe et le monde réel (Saussure 1979: 18). F. de Saussure signale à la fois l'immutabilité et la mutabilité du signe. L'immutabilité, parce que «'arbitraire du signe rend indispensable l'adhérence à la convention, car sans ceci on aboutirait à l'incompréhension totale. De même, la complexité de la langue et le fait qu'elle est une chose dont tous les individus se servent toute la journée la rendent résistante au changement» (ibid.: 32). D'autre part, le temps altère toutes choses; "ce même arbitraire qui militait contre le changement a pour conséquence paradoxale qu'une langue est radicalement impuissante à se défendre contre les facteurs qui déplacent d'instant en instant le rapport du signifié et du signifiant» (la mutabilité). Il faut toutefois remarquer que ce changement linguistique est de nature aveugle et fortuite, car la «langue ne prémédite rien» ibid.: 97).

On peut constater que, dans le système de Saussure, cette argumentation est irréfutable: l'acceptation de l'idée d'un signe arbitraire ne laisse aucune possibilité d'explication linguistique des réformes de la langue. Mais, en dépit de cela, au XX siècle, plusieurs débats sur les langues, ainsi que des tentatives de réforme prennent place. Peut-être doit-on recourir à l'argumentation ayant comme point de départ le caractère non-conventionnel (naturel) des signes linguistiques? À l'instar de É. Benveniste, qui délimite la zone de l'«arbitraire»: «Ce qui est arbitraire, c'est que tel signe, et non tel autre, soit appliqué à tel élément de la réalité, et non à tel autre» (Benveniste 1974: 52). L'arbitraire n'existe ici aussi que par rapport au phénomène ou à l'objet matériel et il n'intervient pas dans la constitution propre du signe. Pour Benveniste, le signifiant et le signifié, l'image acoustique et la représentation mentale, ne sont donc en réalité que les faces d'une même notion. Pour le sujet parlant, il y a entre la langue et la réalité adéquation complète: le signe recouvre et commande la réalité: mieux, il est cette réalité $e^{4}$ On reconnaît donc le caractère motivé des mots, ce qui, comme on peut le présumer, n'exclut pas la possibilité de l'influence consciente sur la langue. Donc, si l'on envisage une langue comme système abstrait, on est obligé d'accepter son "imperméabilitée aux manipulations extérieures, mais on ne peut pas être tellement catégorique en parlant de la langue fonctionnant dans une société.

${ }^{4} \mathrm{D}$ 'où la foi dans le pouvoir magique du verbe: nomen-omen. 


\section{3. LA FONCTION SOCIO-SYMBOLIQUE DE LA LANGUE}

Les réflexions qui précèdent nous incitent à effleurer le problème général des fonctions de langue. Il est hors de notre compétence ici de traiter les différents points de vue sur le nombre de fonctions ainsi que de faire état de leur contenu. Nous estimons néanmoins utile de considérer l'une d'elles, la fonction socio-symbolique (Yavorska 2000: 122). Cette fonction consiste en la manifestation, l'acceptation ou le rejet des relations sociales et des valeurs culturelles qui existent dans une société. La fonction socio-symbolique peut-être réalisée, par l'intermédiaire de moyens phonétiques, grammaticaux et lexicaux, dans la communication personnelle (manifestation d'appartenance à un groupe social), ou elle peut englober toute la société. Comme le fait remarquer la linguiste ukrainienne G. Yavorska (ibid.: 234), la valeur symbolique de certains phénomènes linguistiques devient plus importante (et plus manifeste) pendant les époques de grands bouleversements sociaux. Voici quelques exemples. Après la révolution d'octobre 1917 en Russie, le passage à une nouvelle époque a été symbolisée par l'adoption du nouveau calendrier grégorien, de nouveaux noms de rues, de villes, etc. Les noms historiques ont été abolis, ce qui signifiait la rupture avec le passé (le vieux monde de l'inégalité sociale) ${ }^{5}$.

En ukrainien, dans l'élaboration des principes de l'orthographe des emprunts, il y avait un choix entre les modèles russe et polonais. Mais, au début du régime soviétique, le nouveau pouvoir a imposé l'orientation pro-russe, ce qui devait être le symbole de la solidarité avec le nouvel empire soviétique et de la différenciation avec le monde capitaliste de l'Ouest. En Norvège (on y reviendra plus loin), remplacer les anciennes formes linguistiques, plus prestigieuses, par les formes dialectales était vu comme une façon de réduire le pouvoir d'un groupe qui, par héritage et tradition, possédait le pouvoir politique et social dans la société (Bull 1993: 27). Parfois la valeur symbolique peut être transmise par le sous-système d'une langue (le joual-fierté au Québec, «symbole de la contestation d'une société aliénée et aliénante», Corbeil 1980: 24), ou par une langue même (le choix d'une langue dans la situation de bi- ou plurilinguisme, comme au Canada).

Donc, par l'intermédiaire d'une langue, on peut exercer l'effectif contrôle social, ce qui explique l'intérêt accrû (surtout des politiciens) pour les langues (notamment pour leur maintien ou leur réformation). En outre, c'est souvent

${ }^{5}$ Mentionnons que dans le cas où les reformes linguistiques servent de moyen pour créer un nouveau monde, il existe des conditions favorables à l'usage abusif d'une langue par l'État. La langue du régime totalitaire est un exemple de manipulation totale de la conduite langagière et de la conscience linguistique des locuteurs. C'est à l'aide de la langue qu'on crée une image mythique de la réalité, qui divise très nettement le monđe en «nous» et «eux». De plus ce n'est pas exclusivement par le discours politique que s'exprime la langue du totalitarisme: elle met son sceau sur les autres types de textes: scientifiques, littéraires, etc. 
l'idéologie ${ }^{6}$ (et non pas les arguments linguistiques) qui prévaut dans le discours des politiciens au cours des débats sur les langues et au moment de l'introduction des réformes. L'idéologie se caractérise par les paramètres "spatiaux» ou «temporels». La dimension «spatiale» présuppose la prise de position par rapport aux langues et aux cultures avec lesquelles la langue étudiée est en contact «synchronique». Par contre, la dimension «temporelle» sous-entend ou la préservation des traditions nationales et culturelles, ou la rupture avec le passé. L'acceptation de certains liens et le rejet des autres doivent contribuer à l'acquisition de l'identité par

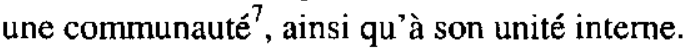

Des projets de réformes linguistiques radicales apparaissent généralement pendant les périodes d'importants changements socio-historiques. Ces réformes affectent surtout l'orthographe, car c'est là que les possibilités de régulation de l'activité linguistique sont les plus évidentes. Par ailleurs, le rôle de l'écriture ne peut pas être réduit complètement aux manipulations politiques, parce qu'elle est un important symbole culturel (cf. C. G. Jung) et une partie essentielle de notre mentalité.

\section{L'AMÉNAGEMENT DU STATUT ET L'AMÉNAGEMENT DU CORPUS}

La signification des symboles est très importante pour les partisans de l'approche sociolinguistique, signalée par T. Bull ${ }^{8}$ (Bull 1993: 31). Pour lui, en aménagement linguistique, vu comme un choix entre des alternatives, l'effet symbolique de ce choix est fondamental. Le perfectionnement de la langue n'est pas la tâche principale': tout au contraire, c'est la langue dans son état actuel qui peut être utilisée pour améliorer la vie sociale, ou pour maintenir le pouvoir de l'État.

Dans l'instrumentalisme (une autre approche dégagée par Bull), une langue est envisagée comme le moyen de communication (fonction principale). La langue standardisée est plus efficace et plus rationnelle qu'une langue non-standarisée.

${ }^{6}$ «L'idéologie est un système global plus ou moins rigourcux de concepts, de mythes, de représentations qui dans une société donnée affirme une hiérarchie de valeurs et vise à modeler lcs comportcments individuels et collectifs» (Monière 1977: 13).

${ }^{7}$ La nécessité de l'identification (de la détermination politique et culturelle) augmente dans les périodes de crises dans la vie d'une société. Cette quête d'identité de l'homme modernc sc traduit aussi dans le sentiment d'appartenance à une nation, qui est «une communauté de gens qui partagent les éléments significatifs profonds de l'héritage commun et le destin commun dans le futur» (Emerson 1960: 95).

${ }^{8}$ Cette classification des idéologies dans l'aménagement linguistiquc contemporain en Norvège, proposée par T. Bull, inclut aussi une approche esthétique (principes de beauté, d'euphonie, d'harmonic de la langue) que nous ne considérons pas ici.

${ }^{9}$ C'est le cas dans l'instrumentalisme. 
La classification des approches de l'aménagement linguistique de T. Bull nous semble correspondre au schéma sociolinguistique général des étapes de l'aménagement linguistique, d'après lequel on distingue l'aménagement du statut: les moyens et les actions nécessaires pour élargir les sphères du fonctionnement d'une langue (ce qui correspond à l'approche sociolinguistique de T. Bull), et l'aménagement du corpus: les actions ayant pour but la normalisation d'une langue, notamment de son orthographe, la terminologie, la préparation des grammaires, etc. (ce qui correspond à l'approche instrumentaliste de T. Bull). Passons à l'analyse plus détaillée de ces étapes.

\subsection{PRINCIPES ET ÉTAPES SUCCESSIVES}

Comme exemple réussi de l'aménagement du statut d'une langue, on évoque souvent le cas du Québec, où les autorités sont parvenus à renforcer le statut du français pour aider les Québécois à «échapper à leur condition de peuple dominé et à participer de plain-pied à la vie économique et industrielle du pays et du continent" (Corbeil 1980: 31). Dans la manière de procéder à l'établissement d'un plan d'organisation linguistique, on fait ressortir les principes suivants:

1. Une connaissance approfondie de la situation linguistique de départ (l'animation, les activités d'enquête, le recueil de l'information, les recherches); une description détaillée de la situation avec le maximum de rigueur méthodologique.

2. La définition des caractéristiques de la «situation cible»: cette démarche implique une relation étroite entre les spécialistes qui ont établi la description et les responsables politiques du pays, car les premiers ont la responsabilité de fournir aux seconds les 10 éléments d'information propres à fonder des choix politiques éclairés; il revient à ces derniers de faire ces choix et de leur donner un statut juridique sous forme de loi, de décret, de règlement, de directive, etc. De plus, il est primordial que ces choix politiques fassent l'objet d'un large consensus, qu'ils reçoivent l'adhésion d'un grand nombre d'usagers et qu'ils représentent véritablement et sans ambiguitté aucune un projet collectif.

3. La mise en point d'une stratégie qui permettra de passer de la situation de départ à la situation cible. En outre, J.-Cl. Corbeil définit les deux grands principes du processus par le biais duquel une langue (ou la variante d'une langue) prédomine ou s'impose comme norme. Le premier est le principe de la globalité: ce sont les communications institutionnalisées telles l'enseignement, l'administration publique, les institutions économiques, les médias d'information ou de communication de masse qui déterminent la prédominance d'une variante ou d'une langue sur les autres; le deuxième, c'est le principe des images: chaque individu construit à l'intérieur de lui-même une certaine image de son être linguistique, l'image qui sera à la base de ses attitudes et de ses comportements (les images les plus importantes sont la langue des premières années de scolarité, celle de l'affichage, de la publicité, des raisons sociales, la langue des mass médias, le vocabulaire et la terminologie). 
Les étapes successives de l'aménagement du corpus, définies par E. Haugen (1966), ont été regroupées selon la forme et selon la fonction (usage) linguistiques.

L' aménagement concernant la forme linguistique.

\section{Sélection de la norme}

Sélectionner une certaine variété de langue comme norme signifie favoriser le groupe qui la parle. S'il y a déjà une élite qui utilise une variété particulière, c'est généralement (mais pas inévitablement) cette variété qui va s'imposer. Quand aucun des groupes linguistiques n'a le statut privilégié, le choix de la langue d'un des groupes va entramer la résistance des autres. Dans d'autres cas, on sera obligé de recourir à la construction du nouveau standard en utilisant les procédés comparatif (quand on construit une langue maternelle hypothétique à partir des dialectes apparentés), archaïsant (on prend comme modèle la langue maternelle existante dans les traditions), statistique (modèle le plus apte à gagner l'acceptation générale), ou de reconnaître des normes multiples (comme en Afrique, par exemple).

\section{Codification de la forme}

L' aménagement linguistique visant un changement dans la codification implique que l'on ait, au préalable, une représentation claire des codifications précédentes et de leurs relations quant à l'usage. C'est pourquoi les recherches linguistiques, essentielles dans tout programme de codification, peuvent être un facteur important de la réussite. L'usage peut être commun sans aucune énonciation formelle des règles, ou, au contraire, les règles fournies par les organismes de législation linguistiques peuvent être entravées par la tradition et l'absence de volonté de les utiliser. Il faut souligner que plus le mot est technique, plus il importe qu'il soit standardisé (c'est ici que les commissions de terminologie peuvent s'avérer les plus efficaces).

L'aménagement quant à la fonction linguistique (c'est-à-dire, l'usage des structures linguistiques).

\section{3. Élaboration de la fonction}

Aujourd'hui, une langue standardisée doit avoir les différences stylistiques pour accomplir de diverses fonctions dans une société. La langue n'est pas exhaustive si elle ne possède pas un large éventail d'expressions relatives à la vie de l'esprit (pour cela, la langue doit puiser dans le langage oral ou dans les dialectes ruraux), aux realia de l'ère technologique, etc. Haugen distingue donc deux voies principales d'enrichissement de la langue, qui sont l'innovation et l'adaptation (des emprunts). 


\section{Acceptation par la communauté}

La volonté d'une communauté d'accepter les innovations linguistiques semble déterminée par la disposition de cette communauté à l'égard de la nécessité d'introduire les nouveautés, d'altérer les programmes scolaires, de changer les habitudes langagières, etc.

\subsection{AMÉNAGEMENT DU CORPUS: LE CAS DE NORVÈGE}

On considère les réformes linguistiques en Norvège être un certain succès en

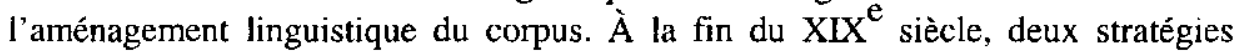
différentes ont été développées afin de donner aux Norvégiens une langue qui pourrait servir comme l'expression unique de leur individualité nationale. La première, promouvant le bokmål («langue livresque»), faisait reposer les changements sur le langage parlé par les classes privilégiées dans les villes et les régions du SudEst du pays (variété du danois). La deuxième favorisait le nynorsk («nouveau norvégien»), ayant pour base les dialectes norvégiens pour la plupart des régions rurales de l'Ouest. Dès les tout premiers débats sur les langues, en 1860 , l'aménagement linguistique a été un sujet de préoccupation politique, les réformes étant réalisées par les autorités officielles. Les comités, mandatés par le gouvernement pour faire le travail préparatoire linguistique, ont été appelés à ne pas faire reposer la langue standard sur le langage de l'élite sociale (ce qui n'était pas commun dans l'histoire de la standardisation). La politique adoptée, consistant à fusionner les deux variétés de langue norvégienne dans le norvégien uni, s'est avéréc être un succès «partiel». Elle a arrêté, même inversé la tendance à la prononciation orthographique (basée sur l'orthographe danoise), en donnant aux deux langues un cadre orthographique norvégien commun, mais l'objectif ultime, celui de fusion des variétés linguistiques, n'a pas été atteint.

Aujourd'hui, en Norvège, il y a deux langues officielles écrites, une situation qui persiste depuis les années 1970. Plus spécialement, en 1993, 83\% des élèves utilisaient le bokmål comme langue principale dans les écoles, le nynorsk n'étant utilisé que par $17 \%$ des élèves. Dans la standardisation du nynorsk, il n'y avait pas d'orientations claires (conservatrices ou radicales), ce qui a contribué à la croissance de la liberté de choix, à la variation ${ }^{10}$, et ce qui a affaibli ses positions par la suite. La réforme de l'orthographe de 1981, par laquelle on promouvait le bokmål comme langue officielle ou standard, signifiait l'abandon des efforts controversés d'imposer un modèle construit par le mélange des deux variétés (Bull 1993: 35). Il s'est avéré que la proclamation du standard par un organisme officiel

${ }^{10} \mathrm{Il}$ faut préciser que cette languc a un système de déclinaison plus compliqué que dans lc bokmail. 
de l'aménagement linguistique, voire par le Parlement, n'est pas suffisante, car les formes linguistiques ne gagnent pas de prestige automatiquement par le processus de la standardisation: on finit parfois par obtenir un hybride que personne ne reconnaît comme sien, et que personne n'utilise par la suite. D'autre part, le triomphe du bokmål peut être un argument en faveur du développement «libre» («naturel») d'une langue, quoique Haugen considère de telles affirmations comme mystiques («in our day there is too much plastic surgery and skin grafting to convince anyone by such arguments», Haugen 1966: 292). On explique la survivance du bokmål par son vocabulaire correspondant aux besoins des temps modernes (précision de l'expression qui en fait le médium efficace de l'échange de l'information, surtout à l'époque de l'information), par sa capacité de résister et de s'accommoder aux changements par des structures bien implantées («well-drilled structures»), par une grande quantité de locuteurs (plus de 80\%, concentrés dans les régions urbaines), par son caractère urbain (surtout a l'ère de l'industrialisation et de l'urbanisation, quand les nouvelles «élites de masses», notamment les hommes d'affaires, manifestent plutot leur appui aux langues considérées comme moyen de promotion sociale qui est le bokmål).Tout cela, renforcé par la règle générale selon laquelle «la langue abhorre la duplication» (Haugen 1966: 288), et face à la schizoglossia (dédoublement de la personnalité qui laisse beaucoup de personnes linguistiquement divisées et incertaines), a favorisé l'élan du bokmål.

Ainsi, d'un côté, les changements sociaux, pour être reflétés par la langue, doivent être «introduits» par un organisme linguistique. De l'autre côté, l'aménagement linguistique, même bien organisé, ne peut pas être considéré comme réussi sans passer par l'étape de l'acceptation par la communauté. Quelle est la solution? Nous ne prétendons pas l'avoir, mais nous essaierons de formuler quelques suggestions dans la partie suivante.

\section{CONCLUSION}

Une Académie de la langue pouvait jouer un rôle important à l'époque d'enthousiasme idéologique et de ferveur nationale. Avec le début de l'ère postmodeme, l'Académie court le risque de «se fossiliser, si elle ne sait pas se réorienter en fonction des problèmes de l'usage actuel de la langue» (Jemudd 1993: 136). D'après B. H. Jemudd, si une langue proposée par une institution officielle va à rencontre de la demande du peuple et de l'usage établi, elle risque de se heurter à une forte résistance de la communauté.

La linguistique du $X X X^{\mathfrak{e}}$ siècle a fourni des informations précieuses sur la nature de la langue qui fonctionne dans une société. À la lumière de ces études, l'approche de "gestion linguistique» (language management approach) de Jemudd nous semble non dépourvue de sens: dans l'aménagement linguistique, le linguiste con- 
state donc la nécessité de réévaluer le rôle de l'intervention gouvernementale. Selon les termes de P. L. Garvin (Garvin 1993: 42), cette orientation est appelée le style d' "imposition académique» (academy-govemed style), par opposition au style de «libre entreprise» (free-enterprise style). La première présuppose l'existence d'une seule source d'autorité incontestée, qui est reconnue et acceptée par tout le monde; la deuxième est caractérisée par l'absence d'un organisme chargé de la formulation et de la diffusion des règles de propriété de la langue (l'établissement et la diffusion de ces règles deviennent une affaire de libre entreprise, car elle est entre les mains des «institutions privées», telles les gens appartenant à une communauté linguistique, les maisons d'édition, y compris des dictionnaires, le système scolaire décentralisé, etc. C'est le jumelage de ces deux approches (surtout dans le cas de l'aménagement linguistique du corpus) qui nous semble le plus apte à répondre aux exigences de notre époque et à donner les résultats les plus satisfaisants dans la régulation des problèmes linguistiques.

\section{BIBLIOGRAPHIE}

Benveniste, E. (1974), Problèmes de linguistique générale, Paris: Gallimard.

Bull, T. (1993), Conflicting ideologies in contemporary Norvegian language planning, dans: Language Conflict and Language Planning, Berlin-New York: Mouton de Gruyter.

Cameron, D. (1990), Demythologizing socioliguistics: why language does not reflect society, dans: ldeologies of Language, London-New York: Routledge.

Cooper, R. L. (1983), Language planning and social change, Cambridge: Cambridge University Presse.

Corbeil, J.-C. (1980), L'aménagement linguistique du Québec, Montréal: Guérin.

DPh, Dictionnaire philosophique [sous la direction de I. Frolov; traduit du russe], (1980), Moscou: Éditions du Progrès.

Dirk, N. B., Eley, G., Orthner, S. B. (1994), Introduction, dans: Culture/Power/History. A reader in contemporary Social Theory, Princeton: Princeton University Press.

Emerson, R. (1960), From empire to nation; the rise of self-assertion fo Asian and African peoples, Boston: Beacon Press.

Foucault, M. (1978), Itistory of Sexuality, vol. 1, Ncw York: Panthćon.

Garvin, P. L. (1993), A conceptual famework for the study of language standardization, dans: International Journal of the Sociology of Language. The Haguc-New York: Walter de Gruyter, $\mathrm{n}^{0} 100 / 101$, pp. 37-54.

Haugen, E. (1966), Language Conflict and Language Planning: the case of modern norvegian, Cambridge: Harvard University Press.

Jernudd, B. H. (1993), Language planning from a management perspective: An interprétation of findings, dans: Language Conflict and Language Planning, Berlin-New York: Mouton de Gruyter.

Jung, C. G. (1989), La vie symbolique: psychologie et vie religieuse, Carl Gustav Jung, Paris: A. Michel. 
Karam, F. X. (1974), Toward a définition of language planning dans: Advances in language planning, The Hague: Mouton, Joshua A. Fishman (éd.), pp. 103-124.

Monière, D. (1977), Le développement des idéologies au Québec, dès origines à nos jours, Montréal: Québec/Amérique.

Saussure, F. de (1979), Cours de linguistique générale, Pans: Hachctte.

Yavorska, G. (2000), Linguistique prescriptive comme discours: Langue, culture, pouvoir, Kiev: Institut de la linguistique de 0. 0. Potebnia. 